\title{
Practice of a GIS education program for junior and senior high school students
}

\author{
Hiroyuki Yamauchi $^{\text {a, }}$, Takashi Oguchi ${ }^{\text {a, }}$, Yasutaka Haneda ${ }^{\text {b }}$ \\ ${ }^{a}$ Centre for Spatial Information Science, The University of Tokyo, Hiroyuki Yamauchi, h.yamauchi@csis.u-tpkyo.ac.jp , Takashi \\ Oguchi,oguchi@csis.u-tpkyo.ac.jp \\ ${ }^{b}$ Department of Natural Environmental Studies, Graduate School of Frontier Sciences, The University of Tokyo, yhaneda@csis.u- \\ tpkyo.ac.jp \\ * Corresponding author
}

Keywords: GIS education, exercise, QGIS

\begin{abstract}
:
We conducted a GIS (Geographic Information Science) education program for junior and senior high school students in August 2018. This program was designed so that students can learn basic knowledge of GIS and methods of mapping within a day. In this program, we firstly lectured the history of maps and various utilizations using GIS. After the lecture, students exercised mapping and spatial analysis by operating QGIS and free open data. The exercise included spatial analysis using DEMs (Digital Elevation Models) such as terrain visualization based on elevation values, slope calculation, and contour line creation.

At the end of the exercise, we conducted a questionnaire survey to understand how the students evaluated the difficulty, understanding and satisfactory levels of the lecture and the exercise, as well as their PC operating skills. They answered each item by selecting one from five levels.

The answers of the students show that most of them enjoyed both the lecture and the exercise. They tended to feel some difficulties with the materials, but their understanding and satisfactory levels were relatively high. Almost all students who found high difficulty in the program had low PC operating skills, indicating that the lack of the skills led to limited understanding of technical terms such as the desktop and folders.
\end{abstract}

